\title{
On nonlinear dynamic of a non-ideal Duffing system with fractional damping
}

\author{
J M Balthazar ${ }^{1}$, A M Tusset ${ }^{2}$, V Piccirillo ${ }^{2}$, A Nabarrete ${ }^{1}$, G Litak ${ }^{3,4}$ and C Oliveira ${ }^{1,5}$ \\ ${ }^{1}$ ITA-Aeronautics Technological Institute, São José dos Campos, SP, Brazil, \\ ${ }^{2}$ UTFPR - Federal University of Technology - Paraná, Ponta Grossa, PR, Brazil, \\ ${ }^{3}$ Lublin University of Technology, Faculty of Mechanical Engineering, Lublin, Poland, \\ ${ }^{4}$ University of Science and Technology, Kraków, Poland \\ ${ }^{5}$ UFGD, Federal University of Grande Dourados, Dourados, MS, Brazil
}

\begin{abstract}
Fractional damping is appearing in different contexts in any systems with memory and hysteresis. Such damping is defined by a fractional derivative term, in contrary to classical viscous damping which takes into account the first order derivative. In this work, we characterize the nonlinear dynamics of a non-ideal Duffing system, with fractional damping using nonlinear dynamical tools. The non-ideal excitation originates from a DC electric motor with limited power supply driving an unbalanced rotating mass. The response of the system is investigated with the voltage as a control parameter. Numerical simulations show the occurrence of regular and non-regular motions, which are investigated via bifurcation diagrams and phase plane portraits.
\end{abstract}

\section{Introduction}

The study of problems that involve the coupling of several systems was explored widely in the last years in function of the change of constructive characteristics of the machines and structures. In this way, some phenomena are observed in dynamical systems composed by supporting structures and rotating machines, where the unbalancing of the rotating parts is the main cause of vibrations. In the study of these systems, for a more realistic formulation one has to consider the action of an energy source with limited power (non-ideal), that is, to consider the influence of the oscillatory system on the driving force and vice versa. Recently a number of non-ideal vibrating systems has been studied, for some examples, see [1-3], besides others. The above mentioned results consider a nonlinear stiffness, but in all of them the damping element of the structure exerts a linear viscous damping force. However, many materials show a behaviour that differs from a linear viscous damper. Different models for viscoelastic behaviour applying the concept of fractional derivatives were discussed [4]. Fractional calculus have been applied in several fields like as physics, chemistry, finances, and bioengineering [5-6]. In Ref. [7], it is shown that the fractional order duffing excited by a ideal system can produce a chaotic attractor. The idea in this work is study the nonlinear dynamic of a non-ideal Duffing system with fractional damping. The figure 1 shows the non-ideal fractional oscillator. The vibrating system under consideration consists of a main structure of mass $m_{1}$, constrained by a linearly viscous element with damping coefficient $\mu$ and a pseudoelastic SMD. The structure is also connected with a non-ideal DC motor, with 
driving rotor of moment of inertia $J_{M}$ and an unbalanced mass $m_{0}$ with eccentricity $r$. The electrical scheme of the DC motor is shown in Fig. 1(b).

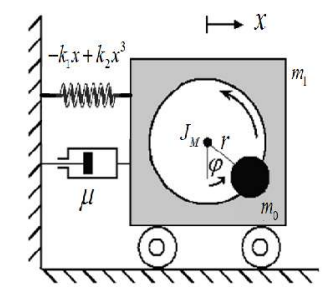

(a)

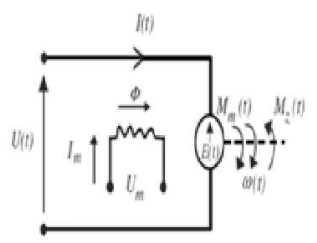

(b)
Figure 1: (a) Non-ideal fractional oscillator and (b) the electrical schematic representation of the DC motor

\section{System description governing equations}

The corresponding equations that govern the angular motion $\varphi$ of the DC motor are typically written in the form [8]. The equations of motion are given by

$$
\left\{\begin{array}{l}
m_{1} x^{\prime \prime}+F_{d}-k_{1} x+k_{2} x^{3}= \\
m_{0} r\left(\left(\varphi^{\prime}\right)^{2} \sin \varphi-\varphi^{\prime \prime} \cos \varphi\right) \\
\left(J+m_{0} r^{2}\right) \varphi^{\prime \prime}=C_{M} \Phi I(t)-m_{0} r x^{\prime \prime} \cos \varphi \\
I^{\prime}=-\frac{R_{t}}{L_{t}} I(t)-\frac{C_{E} \Phi}{L_{t}} \varphi^{\prime}+\frac{U(t)}{L_{t}}
\end{array}\right.
$$

The damping device exerts a force $F_{d}$ given by

$$
F_{d}=c \frac{d^{v} x}{d t^{v}}
$$

and is thus proportional to the vth time derivative of the relative displacement. For $v=1$ the force is a linear viscous damping force and for $v=0$ the force is a linear restoring force. It is assumed that $0 \leq v \leq 1$ and in the interval $0<v<1$ the device contributes with both stiffness and damping. $c$ is the coefficient and has the dimension mass time $v-2$.
It is convenient to work with dimensionless position and time, namely $u=\frac{x}{x_{0}}$ and $\tau=\omega_{0} t$, respectively, where $x_{0}$ is the static displacement, and introducing the following variable $\tilde{I}=\frac{I}{I_{0}}$, where $I_{0}$ is a rated current in the armature.

The non-dimensional fractional derivative is defined as: $\frac{d^{v}}{d t^{v}} \rightarrow \omega_{0}^{v} \frac{d^{v}}{d \tau^{v}}$. In this way is possible to rewrite the Eq. 1 in the dimensionless form:

$$
\left\{\begin{array}{l}
\ddot{u}+\beta(v) u^{(v)}-u+\delta u^{3}= \\
w_{1}\left((\dot{\varphi})^{2} \sin \varphi-\ddot{\varphi} \cos \varphi\right. \\
\ddot{\varphi}=p_{3} \tilde{I}(\tau)-w_{2} \ddot{u} \cos \varphi \\
\dot{\tilde{I}}=-p_{1} \tilde{I}-p_{2} \dot{\varphi}+U_{1}(\tau)
\end{array}\right.
$$

Here the non-dimensional quantities are

$$
\begin{aligned}
& \omega_{0}^{2}=\frac{k_{1}}{m_{1}+m_{0}}, \quad \beta(v)=\frac{c}{m_{1} \omega_{1}^{2-v}}, \\
& \delta=\frac{k_{2}}{k_{1}} x_{0}^{2}, w_{1}=\frac{m_{0} r}{m_{1} x_{0}}, w_{2}=\frac{m_{0} r x_{0}}{\left(J+m_{0} r^{2}\right)}, \\
& p_{1}=\frac{R_{t}}{L_{t} I_{0} \omega_{0}}, U_{1}=\frac{U}{L_{t} I_{0} \omega_{0}}, p_{2}=\frac{C_{E} \Phi}{L_{t} I_{0}} \\
& p_{3}=\frac{C_{M} \Phi I_{0}}{\left(J+m_{0} r^{2}\right) \omega_{0}^{2}} .
\end{aligned}
$$

$\beta(v)$ is a nondimensional coefficient for the damping force, and is possible to note that when $v=1$ the coefficient $\beta(v)$ becomes $\beta(v)=\frac{c}{m_{1} \omega_{1}}$ that is a classical nondimensional coefficient for the damping force.

\section{Numerical Results}

The numerical simulation of the vibrating system was carried out for parameters of the DC motor and mechanical parameters. Numerical dimensionless 
parameters are: $\delta=0.1, w_{1}=0.2$, $w_{2}=0.3, p_{1}=0.3, p_{2}=3, p_{3}=0.15$, $h=\pi / 200$ (integration step).

In order to characterize quantitatively the attractors involved in this study, we computed the 0-1 test proposed by [9]. In the last years, several researches have been applied this test to characterize the attractor of the dynamical system mainly in systems where exist difficulties to calculate the Lyapunov exponents. A complete overview about theoretical aspects and applications of the $0-1$ test can be found in [10].

This test yields a binary type of result: 0 for regular (periodic/quasi periodic) and 1 for chaotic signals. Based on the time series $u$ we define the dimensionless displacements in the $(p, q)$ plane as follows [12]

$$
\begin{aligned}
& p(n, \bar{c})=\sum_{j=0}^{n} u(j) \cos (j \bar{c}) \\
& q(n, \bar{c})=\sum_{j=0}^{n} u(j) \sin (j \bar{c})
\end{aligned}
$$

where $\bar{c}$ is a constant, $\bar{c} \in(0, \pi)$. If the system dynamics is chaotic, the motion in the $(p, q)$ plane would be unbounded, while it would be bounded for regular (periodic/quasiperiodic) motion. The mean square displacement is expressed as [10]

$$
\begin{array}{r}
M(n, \bar{c})=\lim _{N \rightarrow \infty} \frac{1}{N} \sum_{j=1}^{N}\left[(p(j+n, \bar{c})-p(j, \bar{c}))^{2}\right] \\
+\left[(q(j+n, \bar{c})-q(j, \bar{c}))^{2}\right]
\end{array}
$$

For regular dynamics, it is quite likely that $M(n, \bar{c})$ will be a bounded function of $\mathrm{n}$ but not for chaotic dynamics. From this one can define the asymptotic growth rate of the mean square displacement using covariance and variance measures:

$$
K_{\bar{c}}=\frac{\operatorname{cov}(Y, M(\bar{c}))}{\sqrt{\operatorname{var}(Y) \operatorname{var}(M(\bar{c}))}}
$$

$$
\begin{aligned}
& \text { where } \quad Y=\left[1,2, \ldots, n_{\max }\right] \quad \text { and } \\
& M(\bar{c})=\left[M(1, \bar{c}), M(2, \bar{c}), \ldots, M\left(n_{\max }, \bar{c}\right)\right]
\end{aligned}
$$

are vectors. Finally, the median is taken of $K$ - values (Eq. 7) corresponding to 100 different values.

Figure 2 shows the bifurcation diagram for the non-ideal oscillator in terms of the control parameter $U_{1}$. For low values of it we have limit cycle (periodic attractors, see Fig. 3(a) and $\mathrm{K}=0$ ). This attractors suffer an abrupt change at $U_{1} \approx 3.7$, where a bifurcation occurs, and after the bifurcation, there appears chaotic motion (see Fig. 3(b) and $\mathrm{K}=0.99$ )

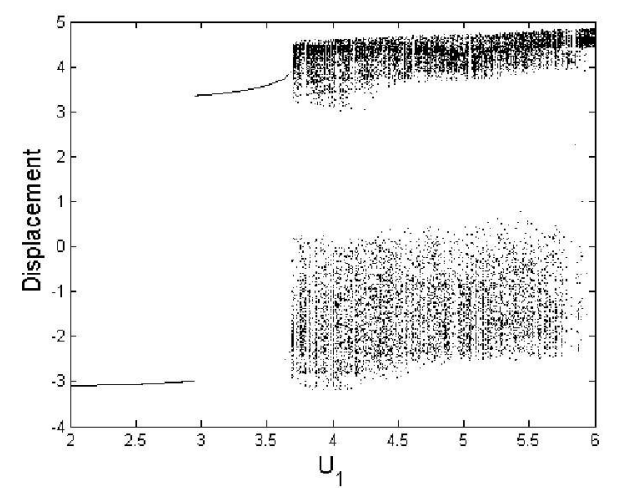

Figure 2: Bifurcation diagram showing displacement as a function of $U_{1}$ and a linear viscous damper $(v=1)$.

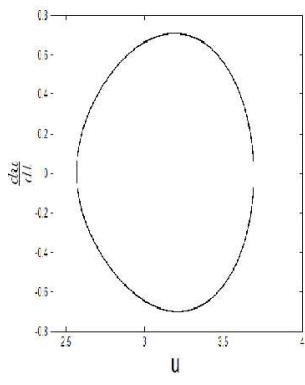

(a)

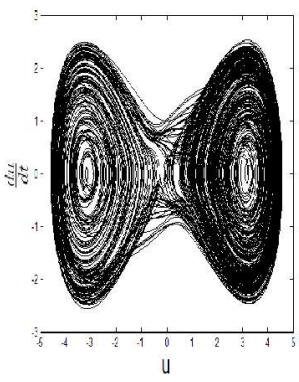

(b)
Figure 3: (a) Phase portraits for $U_{1}=3.6$, and (b) Phase portraits for $U_{1}=3.8$.

Fig. 4 shows the results of the bifurcation diagram of the non-ideal oscillator in terms of the order of the derivative $v$ and $U_{1}=4$ (note chaotic behavior for $v=1)$. For low values of it we have limit cycle (chaotic attractors, see Fig. 5(a) and $K=0.99$ ). This attractors suffer an abrupt change at $v \approx 1.35$, where a bifurcation occurs, and after the bifurcation, 
there appears periodic motion (see Fig. 5(b) and $K=0$ ).

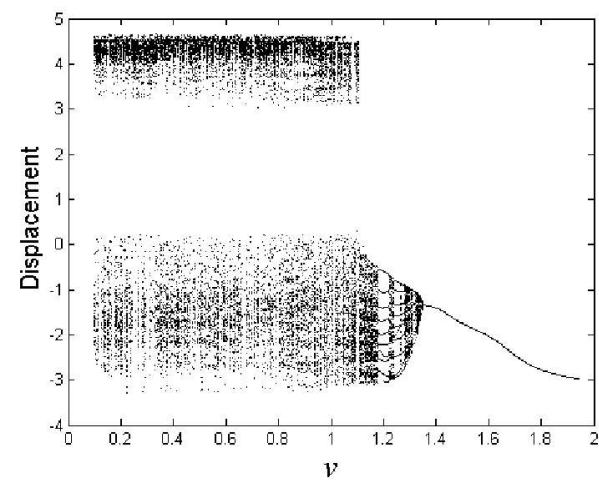

Figure 4: Bifurcation diagram showing displacement as a function of $v$ and $U_{1}=4$

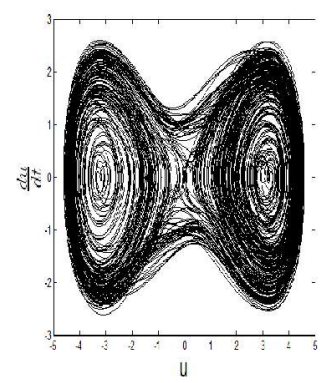

(a)

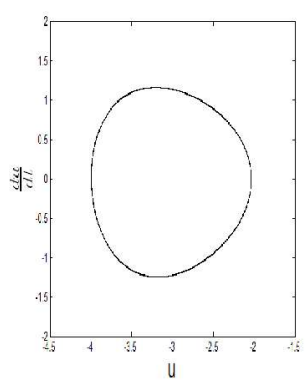

(b)
Figure 5: Phase portraits for $U_{1}=4$ : (a)

$$
v=0.8, \text { and (b) } v=1.6 \text {. }
$$

Figure 6 represents the resonance curve of non-ideal oscillator when $U_{1}$ is slowly increased, and the occurrence of the jump phenomenon during the passage through resonance $U_{1}=3$ is observed only $v=1$.

\section{Conclusions}

In this paper we investigated the dynamics of the fractional order non-ideal Duffing oscillator by phase portraits, $0-1$ test and bifurcation diagrams, and we made a comparison with a non-ideal Duffing oscillator with integer order. When the parameter $U_{1}=4$ the total orders of the system for the existence of chaos are $v<1.35$.

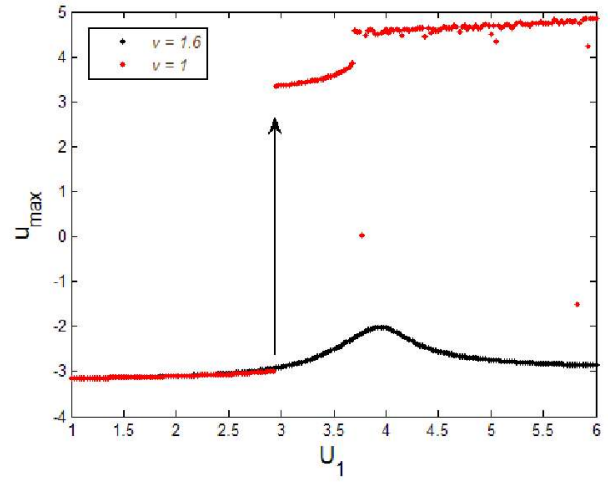

Figure 6: Frequency-response diagram in red $v=1$, and $v=1.6$.

\section{Acknowledgements}

GL is very grateful to prof. J.M. Balthazar and ITA- Aeronautics Technological Institute for hospitality, and also the Polish National Science Center for partial support under the grant agreement No. 2012/05/B/ST8/00080.

\section{References}

1. V. Piccirillo, J.M. Balthazar, A. Tusset, D. Bernardini, G. Rega, Int J NonLinear Mech. 77, 12-27 (2015).

2. V. Piccirillo, J. M. Balthazar, A. Tusset, D. Bernardini, G. Rega, Proc Inst Mech Eng Part C J Mech Eng Sci 230, 120-132 (2016).

3. S.L.T. de Souza, I.L. Caldas, R.L. Viana, J.M. Balthazar, R.M.L.R.F Brasil, Chaos, Solitons \& Fractals 6, 1211-1220 (2005).

4. R.L. Bagley, P.J. Torvik, Journal of Rheology 27, 201 (1983).

5. M. Santhiah, P. Philominath an, M.I. Raja, K. Murali, International Journal of Bifurcation and Chaos 21, 161-175 (2011).

6. O. P. Agrawal, J. A. Te nreiro-Machado, and I. Sabatier (Eds.), Nonlinear Dynamics 38, Springer, Berlin 2004.

7. A. Syta, G. Litak, S. Lenci, M. Scheffler, Chaos 24, 013107 (2014).

8. J. Warminski, J.M. Balthazar, Journal of the Brazilian Society of Mechanical Sciences and Engineering 25, 413-419 (2003).

9. G. Gottwald, I. Melbourne, Proc $R$ Soc Lond A 460, 603-611 (2004).

10. D. Bernardini, G. Litak, Journal of the Brazilian Society of Mechanical Sciences and Engineering doi: 10.1007/s40430-0150453-y (2016) . 